\title{
Herramientas TIC en el aprendizaje en el área de matemática: Caso Escuela PopUp, Piura-Perú
}

\section{ICT tools in learning in the Mathematics area: Case PopUp School, Piura-Peru}

\author{
Cleofé Genoveva Alvites-Huamaní ${ }^{1}$
}

Universidad Alas Peruanas, Perú

\section{Cita recomendada}

Alvites-Huamaní, C. (2017) Herramientas TIC en el aprendizaje en el área de Matemática: Caso Escuela PopUp, Piura-Perú. Hamut'ay, 4 (1), 18-30. Recuperado de: http://dx.doi.org/10.21503/hamu.v4i1.1393

\section{RESUMEN}

En la formación de los estudiantes de educación básica se hace necesario implementar recursos innovadores con apropiación de las Tecnologías de la Información y Comunicación (TIC), en todas las áreas curriculares, más aún en aquellas donde se tiene una problemática marcada, como es el caso de las matemáticas. Por ello, en este estudio se tuvo como hipótesis: "el programa desarrollo mis habilidades en matemáticas con TIC mejora significativamente el aprendizaje en el área de matemática en las estudiantes del tercer grado de primaria de la IE San José de Tarbes” de la Escuela Pop Up, Castilla-Piura. Por lo que se realizó un estudio con diseño cuasiexperimental, con preprueba-posprueba y grupo de control. Se desarrollaron 21 sesiones de aprendizajes para la ejecución del programa en el primer y segundo bimestre del 2016. La muestra fue de tipo no probabilística, con 139 estudiantes del tercer grado de primaria de las secciones A, B, C y D. Para la recolección de datos se aplicó la prueba Desarrollo mis habilidades en matemática con TIC, en dos momentos, al inicio y final de la experimentación. Los resultados del contraste de hipótesis indicaron $\mathrm{p}=.000<.05$, por lo que se concluyó que el programa "Desarrollo mis habilidades en matemática con TIC" mejora el nivel de logro en el área de matemática.

Palabras Clave: Tecnologías, matemáticas, software educativo, aprendizaje-enseñanza.

\section{Abstract}

In the instruction of students from basic education it is necessary to implement innovative resources with appropriation of the Information and Communication Technologies (ICT) in all curricular areas, especially in those where there is a major problem, as is the case of mathematics. Therefore, in

\footnotetext{
1 Doctora en psicología, Directora, asesora y revisora de Tesis a nivel nacional e internacional de diversas universidades. Coordinadora de la División de Investigación y Extensión Científica Tecnológica de la Dirección Universitaria de Educación a Distancia de la Universidad Alas Peruanas, adscrita al Directorio Nacional de Investigadores e Innovadores, miembro del programa Mujeres Científicas del Perú y miembro del grupo Ecosistemas de México, Editor en Jefe de la Revista Hamut'ay. cleoalvitesh@gmail.com, c_alvites@uap.edu.pe
} 
this study, the hypothesis was: "the Developing my Skills in Mathematics with ICT program significantly improves learning in the area of mathematics in students of third grade of school San José de Tarbes" at Pop Up School in Castilla-Piura. In order to compare it, a study with a quasi-experimental design was performed, with a pre-test - posttest and a control group. A total of 21 learning sessions were developed for the program, which were implemented during the first and second terms in 2016. The sample was non-probabilistic, with 139 third-grade students in sections A, B, C and D. For the collection of data, the test Developing my skills in mathematics with ICT, was applied in two different moments, at the beginning and at the end of the experimentation. The hypothesis test results indicated $\mathrm{p}=.000<.05$, so it was concluded that the "Developing my skills in mathematics with ICTs" program improves the level of achievement in the area of mathematics.

Keywords: Technologies, mathematics, educational software, teaching-learning

\section{INTRODUCCIÓN}

Uno de los requisitos en la formación de los estudiantes de educación básica y universitaria es el área de matemáticas; pero a pesar de todos los esfuerzos que realizan las instituciones educativas, la enseñanza de esta área todavía sigue siendo un problema mundial. Esto ha conllevado a un déficit en el desarrollo de las competencias que integran el perfil del estudiante en matemáticas, como lo muestran los resultados obtenidos por todos los países del continente, con relación al Informe del Programa Internacional para la Evaluación de Estudiantes, PISA, por sus siglas en inglés. Son los países asiáticos, europeos y Estados Unidos quienes tienen lugares privilegiados; pero en el caso de América Latina los resultados no son tan óptimos como se quisiera. Según la BBC Mundo (2013, diciembre 3), los países latinoamericanos están entre los últimos puestos. Infobae América (2013, diciembre 2) refiere que existe un retroceso en materia educativa en los últimos tres años en esta parte del continente, a pesar de los esfuerzos que han realizado los gobiernos y la implementación de políticas para la mejora de los resultados en esta área. No obstante, aún están por debajo de los estándares estipulados por la Organización para la Cooperación y el Desarrollo Económicos (OCDE), debido a que ningún país de Latinoamérica ha obtenido los 494 puntos para matemáticas. En el caso de Perú, el problema es más álgido, al haber ocupado el último lugar, como lo indica PISA (2015) los puntajes obtenidos están muy por debajo del promedio. En el caso de matemática fue de 387 puntos. Este problema también se ha visto reflejado en la Institución Educativa San José de Tarbes, con mayor énfasis en el nivel primario, de acuerdo a lo indicado en el diagnóstico del Proyecto Educativo Institucional (PEI). Se destaca que en el tercer grado se han presentado dificultades en la interpretación de los enunciados de los problemas; todavía existe dificultad para el cálculo mental, las secuencias numéricas e interpretación de gráficos de barras están entre las más resaltantes. Por tal razón, como la mencionada Institución Educativa tiene a su disposición la Escuela Pop Up, que cuenta con una variedad de herramientas tecnológicas, se ha considerado ejecutar un proyecto utilizando estas. Por lo que se consideró como objetivo general de la investigación fue "determinar la influencia del programa Desarrollo mis habilidades en matemáticas con TIC en el aprendizaje en el área de Matemática en los estudiantes del tercer grado de primaria de la IE San José de Tarbes, Castilla, en Piura.

\section{Herramientas Tecnológicas de la Información y la Comunicación (TIC)}

La apropiación de las TIC como herramienta de apoyo al proceso de aprendizaje-enseñanza, por parte de los docentes de educación básica, se ha hecho más frecuente debido a la facilidad de uso y al permitirles utilizarla como una estrategia de apoyo en sus actividades curriculares. Esto hace 
que los estudiantes tengan una mayor experiencia real e interactiva, así como una fuente inagotable de información, tal como aseveran Chaves et al. (2015), al sostener que las TIC contribuyen a la construcción de conocimiento por facilitar el acceso a información e incrementar la interactividad y conectividad, que permiten la mediación pedagógica en los contenidos de los cursos.

Ello conlleva a la promoción del aprendizaje mediante el uso de una variedad de herramientas tecnológicas, el desarrollo de las inteligencias múltiples y los estilos de aprendizaje. Por su parte, Calderón et al. (2014) enfatizan que las TIC son todas aquellas herramientas que incluyen la computadora, sistemas audiovisuales, Internet, telefonía y los diversos equipos que se integra en la aplicación en la enseñanza con alguno de ellos. Así también lo refieren Farrońay y Ancaya (2016) al mencionar que las TIC son un instrumento indispensable para las instituciones educativas, pues permiten realizar diversas funciones, como fuente de información multimedia, hipermedial, medio de expresión para la creación e instrumento cognitivo. Para Riveros, Mendoza y Castro (2011, p. 113) las TIC son herramientas y materiales que facilitan el desarrollo de distintas habilidades, estilos y ritmos de aprendizaje por parte de los estudiantes y a los docentes le permiten generar propuestas metodológicas innovadoras y creativas para mejorar la cognición y el proceso de aprendizaje. También refiere, como Ausubel, que la aplicación de la TIC apoya la secuencialidad, claridad y dificultad graduada de la exposición de las tareas de aprendizaje, la cual facilita la retroalimentación, la comprensión y disposición hacia la materia; destaca el uso de la PC como herramienta intelectual para incorporar estrategias pedagógicas para la interacción, atención y amplificación de las experiencias de los estudiantes. Esto es posible de una manera textual, visual, sonora y audiovisual, lo cual ayuda a una presentación diferenciada de los contenidos y, a su vez, a realizar simulaciones de los fenómenos abstractos. Desde una perspectiva mucho más específica, en el aprendizaje de las matemáticas, según Mendoza y Castro (2011, p. 119), hay indicios de que las herramientas TIC intervienen como agentes catalizadores del proce- so de cambio en el aprendizaje- enseñanza de la matemática, debido a que ofrecen múltiples posibilidades para manejar dinámicamente los objetos matemáticos de diferentes sistemas de representación dentro de esquemas interactivos. Esto en razón de que la tecnología abre espacios para que los estudiantes vivan las matemáticas de una manera diferente. Las TIC son utilizadas en el aula para el desarrollo de habilidades y procedimientos para la comprensión de las mismas. Según Villarreal (2012), la aplicación de las tecnologías en el campo matemático ha traído transformaciones en la creación de ambientes en os que se puede vivenciar la matemática como una ciencia experimental, como un laboratorio matemático donde es posible realizar "un ensayo y error educado", pudiendo este permitirlo y a la vez visualizarlo, lo cual sirve para la comprensión matemática. Para Parra y Díaz (2014), la incursión de las TIC como nuevas herramientas pedagógicas en el área de matemáticas genera una transformación socio-cultural concerniente a la práctica pedagógica, por la factibilidad en el uso de software y ambientes virtuales. Asimismo, los entornos computarizados evidencian procesos para representar y manipular el quehacer docente en el área de las matemáticas. Aulaplaneta (2015, septiembre 8) enfatiza que las TIC son beneficiosas para presentar conceptos de forma más visual e interactiva; permiten relacionar las matemáticas con otros aspectos de la vida, al vincular estas con un componente lúdico. Presenta 25 herramientas para enseñar matemáticas con TIC, como se muestra en la figura 1.

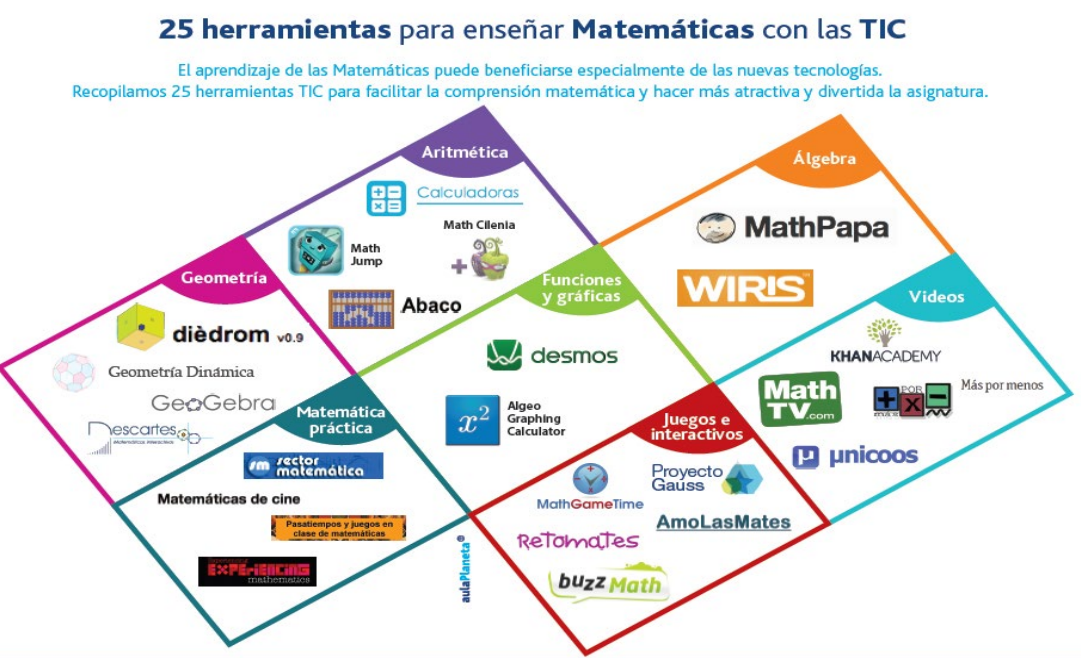

Figura 1. 25 herramientas para enseñar matematicas con TIC.

Fuente: www.aulaplaneta.com 


\section{Aprendizaje en el área de matemáticas}

Según el Ministerio de Educación (2015), el aprendizaje de la matemática debe permitir que los estudiantes desarrollen formas de actuar y pensar matemáticamente en diversas situaciones, que les ayuden a interpretar e intervenir en la realidad a partir de la intuición, el planteamiento de supuestos, formulación de conjeturas e hipótesis para inferir; argumentar para desarrollar métodos $\mathrm{y}$ actitudes útiles para cuantificar ordenar $\mathrm{y}$ medir fenómenos o hechos de la realidad. Márquez y Sánchez (2010) refieren que, de acuerdo a la teoría sociocultural del aprendizaje, se define al aprendizaje como un proceso psicológico de orden superior, el cual se internaliza por las diversas actividades externas que influyen en las estructuras internas, influenciado por instrumentos de orden material denominadas herramientas y de orden psicológico llamados signos. Desde esta perspectiva, el aprendizaje en matemáticas es un proceso donde se sustituyen ideas concretas por otras abstractas; donde los conceptos matemáticos son formales y se aprenden reproduciéndolos, aplicándolos, ejemplificándolos, analizándolos y creando nuevos conceptos; se genera un aprendizaje de procedimientos mediante la construcción progresiva y la puesta en práctica de todo este proceso $y$, en ese sentido, las tecnologías son un medio para afianzar dicho aprendizaje. Por su parte, García (2015) refiere que estos procesos se desarrollan al representar, argumentar, codificar, decodificar, comunicar, calcular, resolver, entre otros; requieren de un tiempo de formación y se van cimentando a lo largo de todo el transcurso de la escolaridad.

Para el Ministerio de Educación (2015, pp.1824), el aprendizaje en el área de matemáticas se da mediante el desarrollo de cuatro competencias.

Competencia 1: Actúa y piensa matemáticamente en situaciones de cantidad. Implica la resolución de problemas de cantidad, en los que se pueda contar y medir para desarrollar el sentido numérico y de magnitud, construir el significado de las operaciones y aplicar las estrategias de cálculo y estimación (figura 2).

Competencia 2: Actúa y piensa matemáticamente en situaciones de regularidad, equivalencia y cambio.

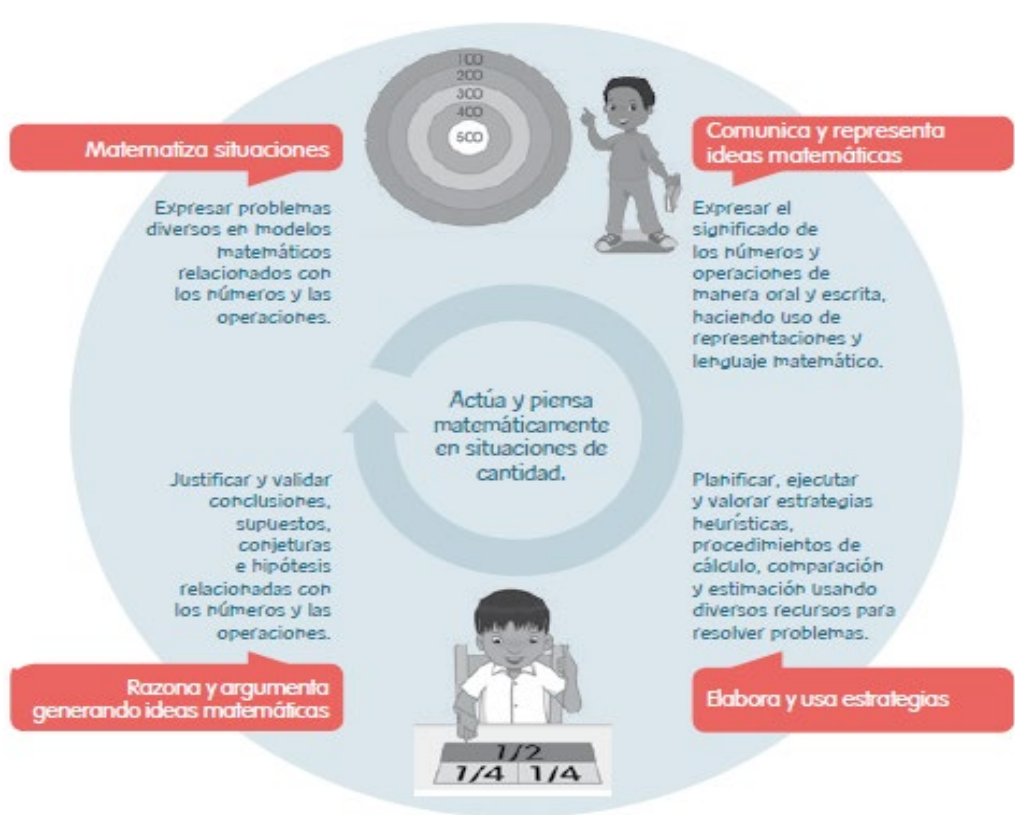

Figura 2. Competencia 1: Actúa y piensa matemáticamente en situaciones de cantidad (Ministerio de Educación, 2015)

Debe desarrollarse progresivamente con la interpretación y generalización de patrones; la comprensión y el uso de igualdades, desigualdades; la comprensión y el uso de relaciones y funciones (figura 3).

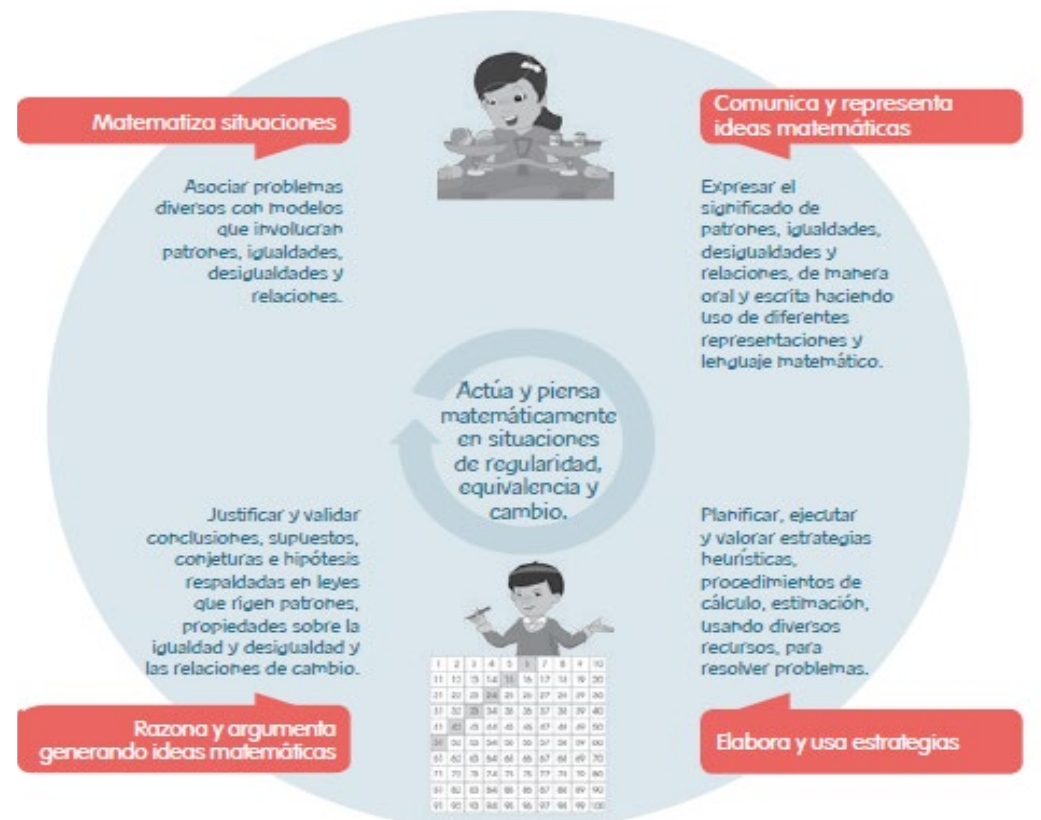

Figura 3. Competencia 2: Actúa y piensa matemáticamente en situaciones de regularidad, equivalencia y cambio (Ministerio de Educación, 2015).

Competencia 3: Actúa y piensa matemáticamente en situaciones de forma, movimiento y localización. Permite desarrollar el sentido de la ubicación en 


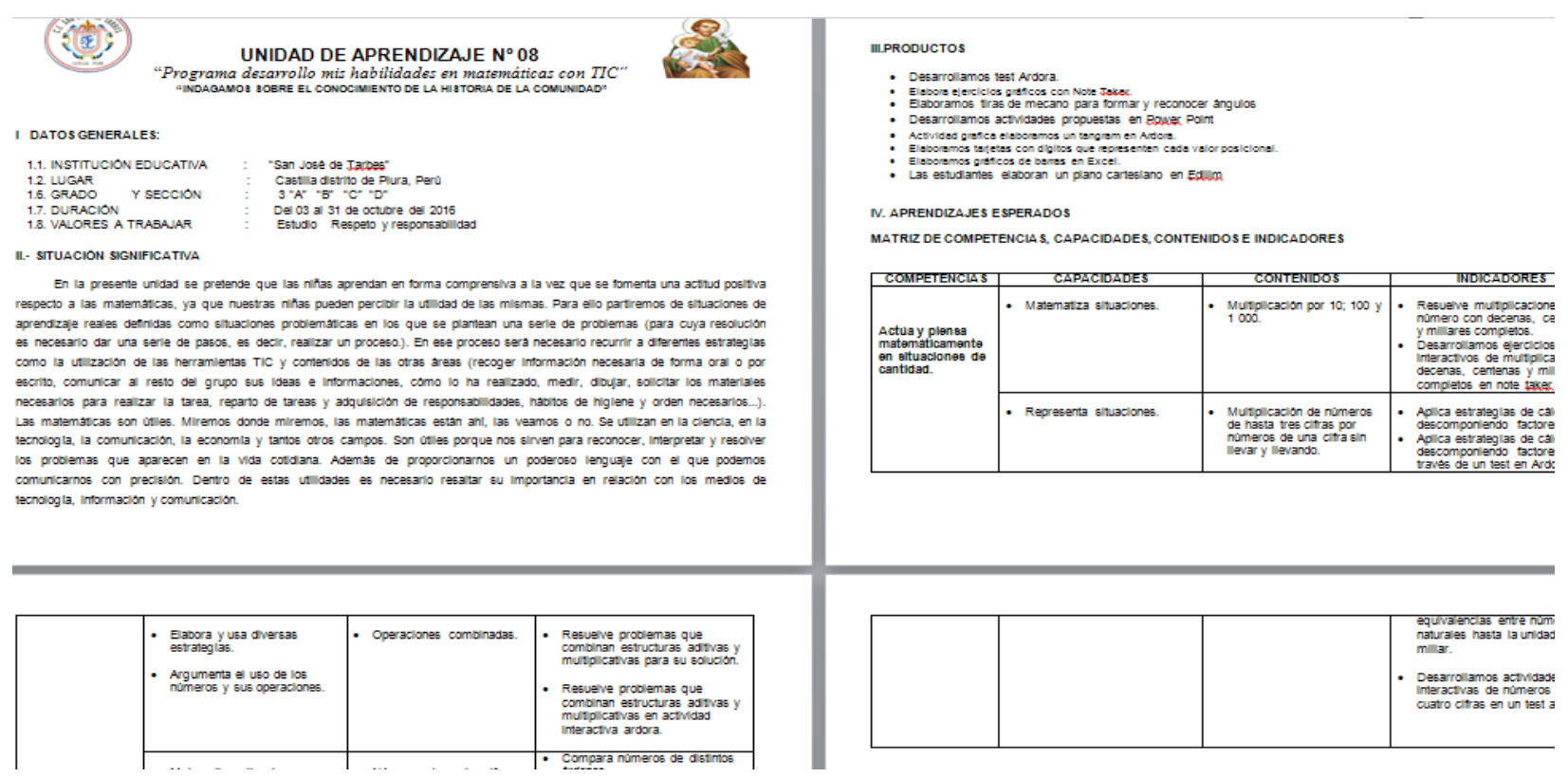

Figura 6. Unidad de aprendizaje elaborada para la investigación.

Fase 3: Elaboración de sesiones de aprendizaje. Las sesiones de aprendizaje constituyen la planificación dosificada y específica de la unidad de aprendizaje e incluyen estrategias y recursos materiales para lograr los aprendizajes propuestos (DCN, 2009, p. 308). El programa experimental estuvo compuesto por 24 sesiones en las cuales se aplicaron las herramientas TIC, como el Software Ardora, note taker, Edilim, Ms Power Point, Excel, entre otros (figura 7).
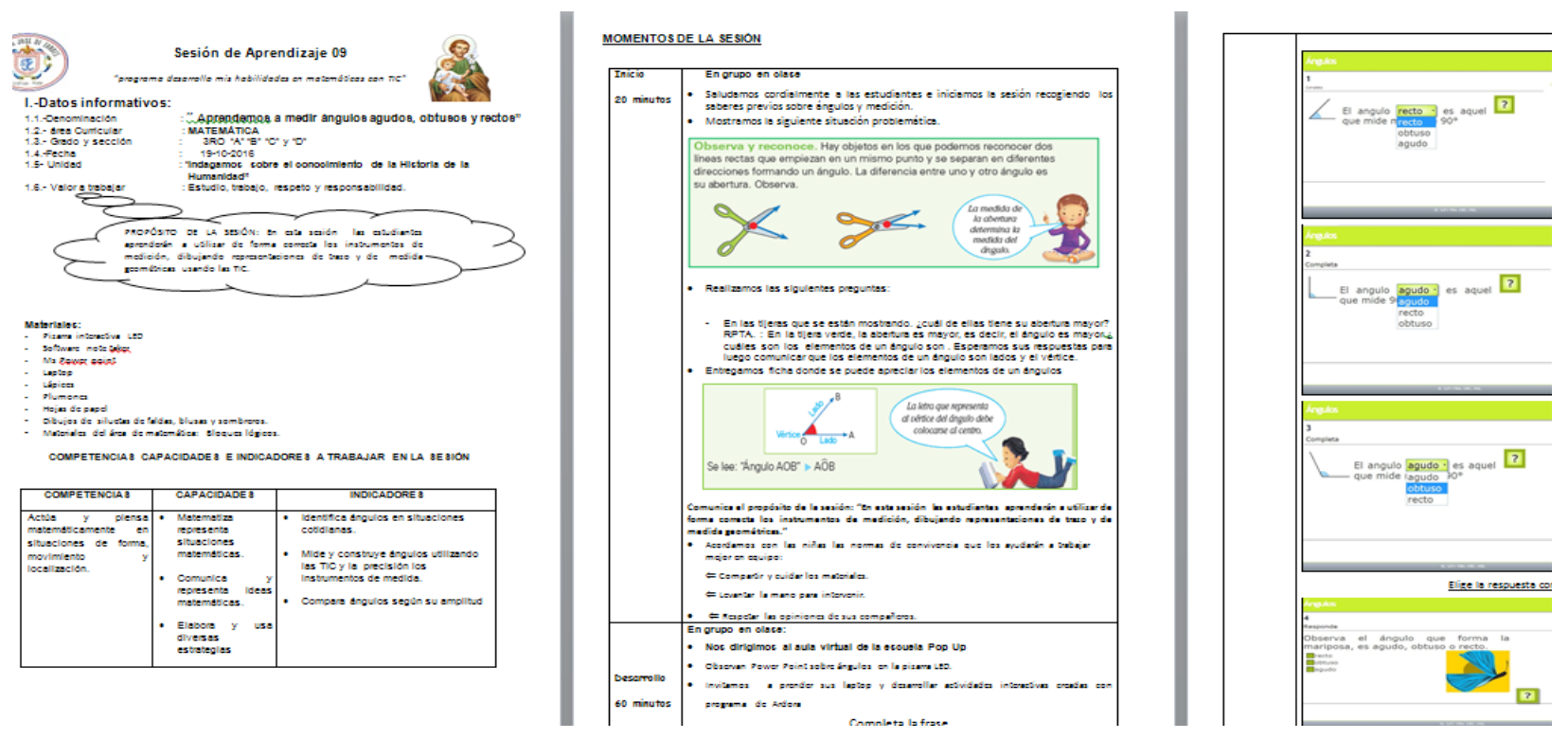

Figura 7. Sesión de aprendizaje elaborada para la investigación, aplicación de Software Ardora. 


\section{Escuelas Pop Up}

La unión de esfuerzos entre el ente público-privado de JP Inspiring Knowledge y Virtual Educa, de manera cooperativa, permitió brindar un nuevo enfoque a las instituciones educativas. Desde su creación, la escuela Pop Up, en Perú, en el año 2015, en el distrito de Castilla, Piura, como parte del plan Ningún niño sin escuela, Ninguna escuela sin conectividad, sirvió para adaptar el aula de clase tradicional a una con características del siglo XXI. De esta manera se acercó a las estudiantes de la educación básica a la apropiación de las TIC, con una infraestructura modular, tecnológica rápida y sencilla, permitiéndose la interacción en múltiples sistemas. Esto permitió incorporar en el proceso de aprendizaje-enseñanza de esta población las herramientas tecnológicas idóneas, contándose con el apoyo de la Fundación Virtual Educa Andina, que propone se siga dando la apropiación continua de estas tecnologías.

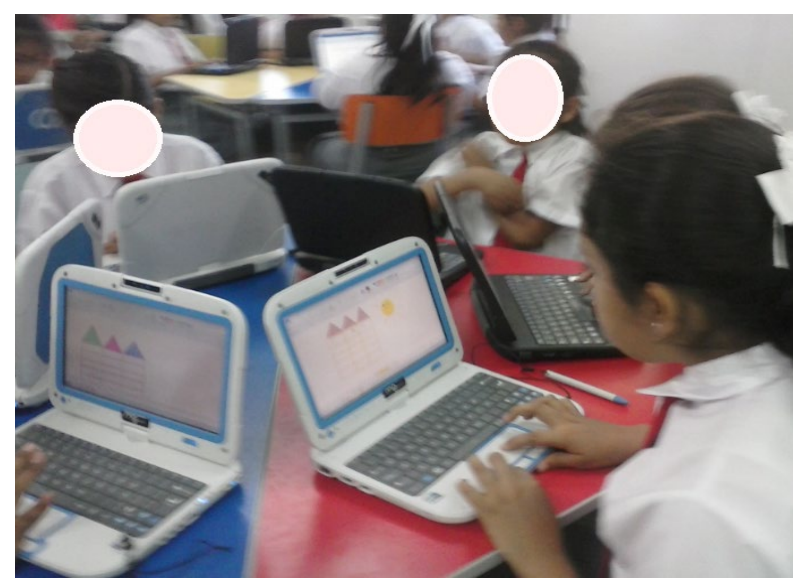

Figura 8. Estudiantes del tercer grado de primaria realizando actividades en la Escuela PopUp. Castilla-Piura.

\section{Materiales y Métodos}

\section{Participantes}

El presente estudio estuvo conformado por una población de 139 estudiantes de cuatro secciones (A, B, C y D) del tercer grado de primaria, que funcionan en la institución educativa San José de Tarbes. Todas las participantes son del sexo femenino, con una edad promedio de entre 8 y 9 años, provenientes de un nivel socioeconómico pobre. La institución educativa está ubicada en la provincia de Castilla, departamento de Piura. La muestra fue de tipo no probabilística. Se trabajó con grupos intactos, ya que en los diseños cuasi-experimentales no se asignan los individuos al azar ni se emparejan los sujetos, grupos están formados antes del experimento (Hernández et al., 2010, p.165.)

Tabla 1

Descripción de la muestra de estudio

\begin{tabular}{lcc}
\hline Grupos & Secciones & Cantidad \\
\hline Control & A y C & 70 \\
\hline Experimental & B y D & 69 \\
\hline & Total & 139 \\
\cline { 2 - 3 }
\end{tabular}

Fuente: I. E. San José de Tarbes.

\section{Instrumento}

Para la recolección de datos se utilizó la prueba Desarrollo mis habilidades en matemática con TIC, descrita en la tabla 2.

La validez del instrumento se determinó mediante juicio de expertos, quienes evaluaron el contenido de la prueba y si esta cumplía con los criterios adecuados para medir las competencias matemáticas. Los especialistas otorgaron una validez promedio de $91 \%$.

La confiabilidad del instrumento se verificó mediante la aplicación de una prueba piloto a un grupo de 20 estudiantes de una institución educativa cercana, la cual tenía características similares a la muestra de estudio. Con los datos de dicha administración piloto se realizó el análisis mediante el coeficiente de confiabilidad de Küder Richardson 20, el mismo que dio un índice de 0.68 , indicando que la prueba era confiable (Me- 
jía, 2005a). También se verificó el grado de dificultad de la prueba, que arrojó 48.79\% (la prueba es relativamente difícil).

Tabla 2

Descripción de la prueba Desarrollo mis habilidades en matemática con TIC

\begin{tabular}{|c|c|}
\hline Dimensiones & Indicadores \\
\hline $\begin{array}{l}\text { Actúa piensa y } \\
\text { matematiza en } \\
\text { situaciones de } \\
\text { cantidad }\end{array}$ & $\begin{array}{l}\text { a. Resuelve multiplicaciones de un número con } \\
\text { decenas, centenas y millares completos. } \\
\text { b. Aplica estrategias de cálculo descompo- } \\
\text { niendo factores. } \\
\text { c. Resuelve problemas que combinan estruc- } \\
\text { turas aditivas y multiplicativas para su solu- } \\
\text { ción. } \\
\text { d. Identifica las equivalencias de números has- } \\
\text { ta cuatro cifras en Unidades de millar, cente- } \\
\text { nas, decenas y unidades. }\end{array}$ \\
\hline $\begin{array}{l}\text { Actúa y piensa } \\
\text { matemáticamen- } \\
\text { te en situaciones } \\
\text { de regularidad, } \\
\text { equivalencia y } \\
\text { cambio }\end{array}$ & $\begin{array}{l}\text { a. Resuelve problemas en los que requiere } \\
\text { realizar el conteo de figuras. } \\
\text { b. Identifica las figuras que completa una se- } \\
\text { cuencia gráfica }\end{array}$ \\
\hline $\begin{array}{l}\text { Actúa y piensa } \\
\text { matemáticamen- } \\
\text { te en situacio- } \\
\text { nes de forma, } \\
\text { movimiento y } \\
\text { localización. }\end{array}$ & $\begin{array}{l}\text { a. Resuelve problemas sobre áreas. } \\
\text { b. Identifica ángulos en situaciones cotidianas } \\
\text { c. Representa mediante pares ordenados la } \\
\text { ubicación de objetos en el plano cartesiano }\end{array}$ \\
\hline $\begin{array}{l}\text { Actúa y piensa } \\
\text { matemáticamen- } \\
\text { te en situaciones } \\
\text { de gestión de } \\
\text { datos e incerti- } \\
\text { dumbre. }\end{array}$ & $\begin{array}{l}\text { a. Emplea tablas y gráficos estadísticos para } \\
\text { analizar e interpretar la información }\end{array}$ \\
\hline
\end{tabular}

Fuente: Fuente: Elaboración propia (2017).

La prueba permite conocer los niveles de logro de los estudiantes en el área de matemática, de acuerdo con cuatro criterios, según lo normado por el Ministerio de Educación (Ministerio de Educación, 2005, p.10; 2009, p. 309), tal como se observa en la tabla 3 .

Tabla 3

Niveles de evaluación de la prueba

\begin{tabular}{lcl}
\hline Nivel & Criterios de evaluación & \multicolumn{1}{c}{ Calificación } \\
\hline 4 & AD & Logro destacado \\
\hline 3 & A & Logro Previsto \\
\hline 2 & B & En Proceso \\
\hline 1 & C & inicio \\
\hline
\end{tabular}

Fuente: Elaboración Propia.

\section{Tipo y diseño}

El estudio es de tipo tecnológico. Los estudios tecnológicos abordan problemas técnicos $\mathrm{y}$, por tanto, pretenden demostrar la utilidad y validez de los recursos tecnológicos en la transformación de la realidad o en la solución de un problema (Sánchez y Reyes, 2006). En tal sentido, el estudio tuvo por finalidad mejorar el aprendizaje de los estudiantes en el área de Matemáticas mediante el uso de las herramientas TIC de la Escuela Pop Up, Piura, Perú (Mejía, 2005a, p. 33). Se utilizó un diseño cuasi-experimental, con preprueba-posprueba y grupo de control. Según Hernández et al. (2010), en este diseño se aplica una preprueba a los grupos de control y experimental, a continuación, se les da estímulo o tratamiento con un programa experimental y, concluido este, se administra la posprueba para, posteriormente, verificar estadísticamente si hay o no diferencias significativas entre ambas mediciones. La tabla 4 describe el modelo del diseño cuasi-experimental.

Tabla 4

Descripción del diseño de estudio

\begin{tabular}{cccc}
\hline \multicolumn{4}{c}{ Diseño cuasi- experimental } \\
\hline$G_{1}$ & $O_{1}$ & $X$ & $O_{2}$ \\
\hline$G$ & $O_{3}$ & - & $O_{4}$ \\
\hline
\end{tabular}

Donde:

$\mathrm{G}_{1}$ : Grupo experimental

$\mathrm{O}_{1}$ : Preprueba

$\mathrm{O}_{2}$ : Posprueba

X: Tratamiento o estímulo con la variable herramientas TIC "Programa desarrollo mis habilidades en matemáticas con TIC”.

G: Grupo de control

$\mathrm{O}_{3}$ : Preprueba

$\mathrm{O}_{4}$ : Posprueba

- : Ausencia de tratamiento o estímulo

Las variables del estudio son:

- Variable independiente (X): Herramientas Tecnológicas de la Información y la Comunicación (TIC).

- Variable dependiente (Y): Aprendizaje en el área de Matemática.

\section{Confidencialidad}

Para garantizar la reserva de la información proporcionada por la muestra, primero se solicitó el consentimiento a la Dirección de la institución 
educativa, la misma que se encargó de solicitar la autorización respectiva a las personas a cargo de las menores. Asimismo, se garantizó la protección de la identidad de la muestra de estudio, en respeto a sus derechos individuales.

\section{Procedimiento}

Para viabilizar el presente estudio, se hizo una serie de coordinaciones con las autoridades de la Institución Educativa San José de Tarbes. Esta institución tiene como parte de su infraestructura a la Escuela PopUp, donde se realizó la investigación y que cuenta con las herramientas tecnológicas idóneas para el desarrollo del trabajo planificado en la forma que se describe a continuación:

Proceso 1. Se solicitó a la Dirección el permiso para que la Fundación Virtual Educa Andina y la Universidad Alas Peruanas puedan ejecutar la investigación en las instalaciones de la Escuela Pop Up. La petición fue aceptada.

Proceso 2. Se coordinó con los docentes responsables de las aulas del tercer grado de primaria, secciones A, B, C y D, para el desarrollo de los contenidos mediante las sesiones de aprendizaje del programa Desarrollo mis habilidades en matemáticas con TIC”. En el trabajo se incluyeron las cuatro secciones.

Proceso 3. Se ejecutó el estudio en el primer y segundo bimestre del 2016. Comprendió la recolección de información mediante la administración de la preprueba a las cuatro secciones y la aplicación del programa solo al grupo experimental, mediante las 24 sesiones de aprendizaje en las que se incluyó el uso de las herramientas tecnológicas.

Proceso 4. Se recolectó datos al concluir la aplicación del programa con la posprueba a las cuatro secciones, para conocer si el programa mejoró el logro de aprendizaje de los estudiantes en el área de matemática.

Proceso 5: Se analizó la información mediante la estadística descriptiva e inferencial. Se utilizó la hoja de cálculo de Excel v.2010 y SPSS v.21.

\section{Resultados}

En el análisis de los resultados se describe en un primer momento los niveles de logro en el área de matemática, de forma general; luego se hace la comparación entre los grupos de control y experimental. En los acápites siguientes se detallan los hallazgos.

\section{Aprendizaje en el área de Matemática}

Según la tabla 5, en el pre test la mayoría de niñas del grupo de control (89.9\%) y del grupo experimental $(92.6 \%)$ se encontraban en inicio. En el post test, la mayoría de estudiantes del grupo de control (50.7\%) se encontraban en proceso, mientras que en el grupo experimental el $44.1 \%$ alcanzó el logro previsto y el $11.8 \%$ el logro destacado.

Tabla 5

Aprendizaje en el área de Matemática según niveles de logro, pre test y post test

\begin{tabular}{llcccc}
\hline & & \multicolumn{2}{c}{$\begin{array}{c}\text { G. de } \\
\text { control }\end{array}$} & \multicolumn{2}{c}{$\begin{array}{c}\text { G. experi- } \\
\text { mental }\end{array}$} \\
\cline { 3 - 6 } Fase & Nivel de logro & $f$ & $\%$ & $F$ & $\%$ \\
\hline Pre test & C (Inicio) & 62 & 89.9 & 63 & 92.6 \\
\hline \multirow{3}{*}{ B (En proceso) } & 7 & 10.1 & 5 & 7.4 \\
\hline & A (Logro previsto) & 0 & .0 & 0 & .0 \\
\hline & AD (Logro destacado) & 0 & .0 & 0 & .0 \\
\hline Post test & C (Inicio & 12 & 17.4 & 3 & 4.4 \\
\hline & B (En proceso) & 35 & 50.7 & 27 & 39.7 \\
\hline & A (Logro previsto) & 22 & 31.9 & 30 & 44.1 \\
\hline & AD (Logro destacado) & 0 & .0 & 8 & 11.8 \\
\hline & TOTAL & 69 & 100.0 & 68 & 100.0 \\
\hline
\end{tabular}

Fuente: Base de datos de los resultados de la prueba de matemática..

\section{Nivel de logro en el área de Matemática por} secciones

De acuerdo con los resultados que se muestran en la tabla 6, en el nivel C (Inicio), el mayor porcentaje de nińas estuvo en tercer grado C (28.6\%); en el nivel B (En proceso), los mayores porcentajes de niñas corresponden a las aulas de tercer grado A (65.7\%) y tercer grado D (44.1\%); en el nivel A (Logro previsto), el mayor porcentaje de niñas 
se registró en tercer grado $\mathrm{B}(42.8 \%)$ y tercer grado $\mathrm{D}(44.1 \%)$ y en el nivel $\mathrm{AD}$ (Logro destacado, el mayor porcentaje de niñas fue en tercer grado $\mathrm{B}$ (14.3\%) y en tercer grado D (11.8\%).

\section{Tabla 6}

Aprendizaje en el área de Matemática, según niveles de logro por secciones

\begin{tabular}{|c|c|c|c|}
\hline Sección & Niveles de logro & $\mathrm{F}$ & $\%$ \\
\hline \multirow{4}{*}{$\begin{array}{l}\text { 3ro. A } \\
n=35\end{array}$} & C (Inicio) & 2 & 5.7 \\
\hline & B (En proceso) & 23 & 65.7 \\
\hline & A ( Logro previsto) & 10 & 28.6 \\
\hline & AD (Logro destacado) & 0 & .0 \\
\hline \multirow{4}{*}{$\begin{array}{l}\text { 3ro. B } \\
n=34\end{array}$} & C (Inicio) & 3 & 8.6 \\
\hline & B (En proceso) & 12 & 34.3 \\
\hline & A ( Logro previsto) & 15 & 42.8 \\
\hline & AD (Logro destacado) & 5 & 14.3 \\
\hline \multirow{4}{*}{$\begin{array}{l}\text { 3ro. C } \\
n=34\end{array}$} & C (Inicio) & 10 & 28.6 \\
\hline & B (En proceso) & 13 & 37.1 \\
\hline & A ( Logro previsto) & 12 & 34.3 \\
\hline & AD (Logro destacado) & 0 & .0 \\
\hline \multirow{4}{*}{$\begin{array}{l}\text { 3ro. } \\
n=34\end{array}$} & C (Inicio) & 0 & .0 \\
\hline & B (En proceso) & 15 & 44.1 \\
\hline & A ( Logro previsto) & 16 & 44.1 \\
\hline & AD (Logro destacado) & 4 & 11.8 \\
\hline Total & & 139 & 100.0 \\
\hline
\end{tabular}

Fuente: Base de datos de los resultados de la prueba de matemática.

La comparación gráfica de los resultados observada en la figura 9, muestra que en tercer grado A es muy evidente que las niñas se hallan en proceso (65.7\%) para desarrollar sus competencias matemáticas; en tercer grado $\mathrm{B}$, un porcentaje acumulado de $57.1 \%$ (la mayoría) están entre el logro previsto y el logro destacado; en tercer grado $\mathrm{C}$ están en transición del proceso $(37.1 \%)$ hacia el logro previsto $(34.3 \%)$ y en tercer grado $\mathrm{D}$, un porcentaje acumulado de $55.9 \%$ están entre los niveles de logro previsto y logro destacado.

Prueba de hipótesis. Para contrastar la hipótesis general, en primer lugar, se determinó si los datos de los grupos de control y experimental cumplían con una distribución normal. Este análisis se realizó a través de la prueba de Shapiro-Wilk, la cual arrojo que todos los datos de ambos grupos, tanto en pre test como en post test, provienen de una distribución normal ( $\mathrm{p}>.05$ ), como se describe en la tabla 7.

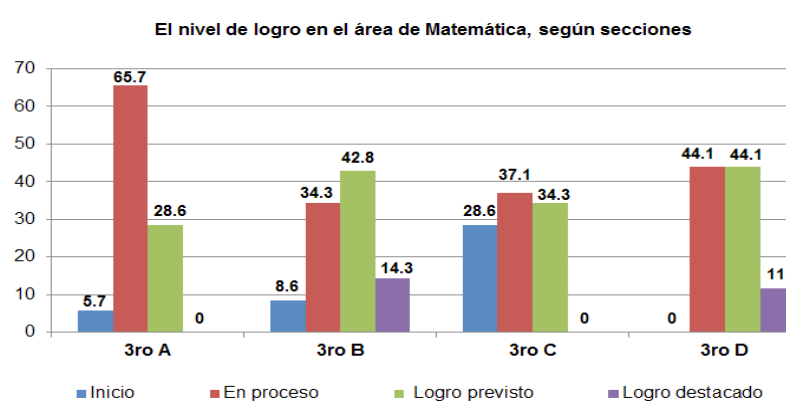

Figura 9. Comparación gráfica del aprendizaje en el área de Matemática, según logro por secciones.

Tabla 7

Prueba de normalidad de los grupos de control y experimental, pre test - post test

\begin{tabular}{llcr}
\hline Fase & Grupos & Shapiro-Wilk & p-valor \\
\hline \multirow{2}{*}{ Pre test } & De Control & .980 & .354 \\
\cline { 2 - 4 } & Experimental & .985 & .565 \\
\hline \multirow{2}{*}{ Post test } & De Control & .974 & .167 \\
\cline { 2 - 4 } & Experimental & .975 & .191 \\
\hline
\end{tabular}

Fuente: Base de datos de los resultados de la prueba de matemática.

\section{Hipótesis general.}

Ha. El programa desarrollo mis habilidades en matemáticas con TIC mejora significativamente el aprendizaje en el área de matemática en los estudiantes del tercer grado de primaria de la IE San José de Tarbes.

H0. El programa desarrollo mis habilidades en matemáticas con TIC no mejora significativamente el aprendizaje en el área de matemática en los estudiantes del tercer grado de primaria de la IE San José de Tarbes.

Para el contraste de la hipótesis general se realizó el análisis inferencial utilizando la prueba $\mathrm{T}$ de Student para muestras independientes, que calculó $\mathrm{p}=.027<.05$ para la diferencia entre los grupos de control y experimental, en el pre test de la prueba de Matemática; es decir, había diferencias significativas entre estos. De igual manera, en el post test, la prueba calculó $\mathrm{p}=.000<.05$, indicando una diferencia significativa entre ambos grupos (tabla 8). 
Tabla 8

Resultado del contraste de hipótesis mediante la prueba de T de Student, pre test-post test

\begin{tabular}{llrrrr}
\hline Fase & Grupos & Media & $\begin{array}{l}\text { Dife- } \\
\text { rencia }\end{array}$ & $\begin{array}{l}\text { T de } \\
\text { Student }\end{array}$ & p-valor \\
\hline Pre test & De Control & 38.65 & 4.43 & 2.243 & .027 \\
\cline { 2 - 6 } & Experimental & 34.22 & & & \\
\hline Post test & De Control & 63.28 & 8.68 & -4.224 & .000 \\
\cline { 2 - 6 } & Experimental & 71.96 & & & \\
\hline
\end{tabular}

Fuente: Base de datos de los resultados de la prueba de matemática.

La influencia de las herramientas TIC en el aprendizaje en el área de Matemática

La figura 10, que se basa en los puntajes totales de la prueba, representa el efecto del programa Desarrollo mis habilidades en matemáticas con TIC. Se observa que, en el pre test, la mediana del grupo de control (39) fue 4 puntos más que la mediana del grupo experimental (35). En cambio, en el post test, como consecuencia del programa, la mediana de los datos del grupo experimental (71) fue 8 puntos más que la mediana del grupo de control (63).

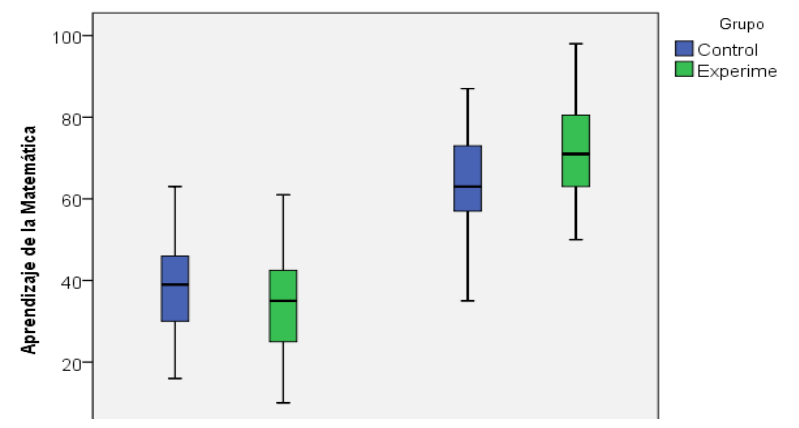

Figura 10. Diagrama de caja y bigotes para la comparación de resultados de los grupos de control y experimental, pre test $\mathrm{y}$ post test.

\section{Discusión y Conclusiones}

El área de Matemáticas es primordial en la educación a nivel mundial; sirve de base para que los estudiantes en formación adquieran y desarrollen competencias idóneas que les permitan continuar desarrollándose académicamente a nivel superior. Pese a su transcendencia, esta todavía sigue siendo un problema para los estudiantes, aun cuando las instituciones de educación básica y superior se han preocupado en buscar estrategias diversas para apalear las dificultades que se presentan en la enseñanza de las mismas; razón por la cual el área de Matemática se tuvo en cuenta en esta investigación.

Los resultados del pre test indicaron que no había diferencias estadísticamente significativas entre el grupo de control (89.9\%) y el grupo experimental (92.6\%), que se encontraban en el nivel C (Inicio). Sin embargo, después de la experimentación con el programa Desarrollo mis habilidades en matemáticas con TIC, con apoyo de la infraestructura de la Escuela Pop Up, se verificaron resultados diferenciados; un porcentaje acumulado de $57.1 \%$ (la mayoría) de estudiantes del grupo experimental del tercer grado B estuvieron entre el logro previsto y el logro destacado; mientras que un porcentaje acumulado de $55.9 \%$ de estudiantes de tercer grado $\mathrm{D}$ estuvieron entre los niveles de logro previsto y logro destacado. Esto indicó la tendencia que las TIC tienen en la actualidad; son relevantes en los estudiantes como herramientas de apoyo en su proceso de aprendizaje. Tales resultados coinciden con los hallazgos de Jame, Zabal y Minguillon (2013), quienes refieren que los estudiantes acogen favorablemente las actividades elaboradas con tecnologías, porque les resulta sencillo el manejo y los contenidos brindados le han sido de gran ayuda en el aprendizaje de matemáticas. En igual sentido se pronuncia Gonzáles (s.f.), al enfatizar que, en el aula, las TIC proporcionan a los estudiantes nuevas formas de aprender las matemáticas. Las utilizan como otro medio para explicar las clases de matemáticas, a través de Paint, software educativo, pizarra digital, lo cual favorece la ampliación de conocimientos y la conexión de ideas y conceptos en la enseñanza de matemáticas en la educación primaria. 
La irrupción de las TIC en el aula ha tenido un avance vertiginoso en todas las áreas y niveles de educación, sobre todo si se tiene en cuenta que los estudiantes de este siglo están habituados a la apropiación de estas, por ser estas herramientas de su generación con las que viven en su vida cotidiana y se interrelacionan con sus pares y su entorno en general.

También se ha podido contrastar en este estudio que el programa Desarrollo mis habilidades en matemáticas con TIC mejora significativamente el nivel de logro en el área de matemática en los estudiantes del tercer grado de primaria de la IE San José de Tarbes, Escuela Pop Up, Piura, Perú $(\mathrm{p}<.05,=.000)$. Estos resultados se relacionan con lo mencionado por González (s.f.) al indicar que las herramientas TIC generan un alto nivel de motivación al aprendizaje de las matemáticas en los estudiantes. Maz-Machado (2012) señala que las matemáticas se han beneficiado de las TIC, al tener una variedad de software que permiten mejorar los procesos de visualización de conceptos; aseguran una adecuada comprensión de ellos al ofrecer variados sistema de representación, como la hoja de cálculo de Excel permite hacer representaciones de diversa índole.

Igualmente, se comprobó en esta investigación que la apropiación de las TIC por medio de la Escuela Pop Up brinda los elementos idóneos para que los estudiantes interactúen con estos nuevos entornos. Hubo una diferencia significativa entre ambos grupos; la mayoría de estudiantes del grupo de control (50.7\%) se encontraban en proceso, mientras que en el grupo experimental el $44.1 \%$ alcanzó el logro previsto y el $11.8 \%$ el logro destacado. Tal resultado coincide con los hallazgos de Vidaurre y Vallejos (2015), quienes refieren que el aprendizaje de la matemática después de la aplicación del software EdiLIM genera en los estudiantes un nivel de logro significativo. Los estudiantes con quienes realizaron el estudio estuvieron en el nivel deficiente y al final del experimento migraron a las categorías regular, bueno y muy bueno, con porcentajes de 10\%, $86.7 \%$ y $3.3 \%$, respectivamente.

\section{Agradecimiento}

De manera especial se agradece a la Hna. Carla Rosales Díaz, Directora de la IE San José de Tarbes; a las docentes María Antonieta Vegas Timoteo, Catherine Paola Rodríguez Coronado, Clara Soledad del Valle Cerna, Heidy Lourdes Sinche Espinoza, Edita Urbina Barreto y Fiorella Rosillo, al haber ejecutado las sesiones de aprendizaje del presente estudio con sus estudiantes del tercer grado de primaria, y a todo el personal técnico que brindó su apoyo en la Escuela Pop Up.

El agradecimiento también a la Fundación Virtual Educa Andina, que ha financiado el presente estudio en aras de una mejora en las competencias de las estudiantes del nivel primario en el área de matemáticas de la Escuela Pop Up, en Piura, Perú.

\section{REFERENCIAS Bibliográficas}

Aulaplaneta. (2015, septiembre 8). 25 herramientas para enseñar Matemáticas con las TIC. Recuperado de: http:// www.aulaplaneta.com/2015/09/08/recursos-tic/25-herramientas-para-ensenar-matematicas-con-las-ticl

BBC Mundo. (2013, diciembre 3). ¿Cómo les fue a los países de América Latina en la Prueba Pisa? Recuperado de http://www.bbc.com/mundo/noticias/2013/12/131203_ pisa_resultados_am

Benavidez, V. (2010). Las evaluaciones de logros educativos y su relación con la calidad de la educación. Revista Iberoamericana de educación, 53, 83-96.

Caro, L., Vargas, T. y Campo, J. (2015). Estudio preliminar sobre el uso de tecnologías de información y comunicación en instituciones de educación superior de la Red Ilumno. Revista Hamut'ay, 2 (1) 49-62. Recuperado de https://doi. org/10.21503/hamu.v2i1.829

Canós-Ruis, N. y Guitert-Cateses, M. (2014). Uso de las TIC en la interacción profesor-alumno: un estudio de caso en una Escuela de Arte y Superior de Diseño. Revista Latinoamericana de Tecnología Educativa, 13 (1) 63-74.

Chaves, O., Chaves, L. y Rojas, D. (2015). La realidad del uso de las TIC y su mediación pedagógica para enriquecer las clases de inglés. Revista Ensayos Pedagógicos, 10 (1), 159-183.

Calderon, S., Nuñez, P. Di Laccio, J. Iannelli, L. y Gil, S. (2014). Aulas-laboratorios de bajo costo, usando TIC. Revista Eureka sobre enseñanza y divulgación de las Ciencias, 12 (1) 212-226.

Ministerio de Educación. (2009). DCN-Diseño Curricular Nacional. Recuperado de: www.minedu.gob.pe/DeInteres/ 
xtras/download.php?link=dcn_2009.pdf

PISA (2015) PISA: Resultados clave. OCDE. Recuperado de: http://www.oecd.org/pisa/pisa-2015-results-in-focusESP.pdf

Farroñay, P. y Ancaya, M. (2016). Gestión administrativa y conocimiento de las TIC en docentes de educación primaria de las instituciones educativas Innova Schools de San Juan de Lurigacho y Ate. Revista Hamut'ay, 3 (1) 35-45. Recuperado de: https://doi.org/10.21503/hamu.v3i1.998

González, L. (s.f.). El conocimiento y uso de estrategias metodológicas apoyadas en las TIC para mejorar el proceso de enseñanza-aprendizaje del área de matemáticas en la educación primaria.

García, B. (2015). Competencias matemáticas, expectativas de aprendizaje y enculturación matemática. Escenarios, 13 (1), 22-33. Recuperado de: https://doi.org/10.15665/esc. v13i1.549

Hernández, R., Fernández, C. y Baptista, P. (2010). Metodología de la investigación (5ta. Ed.) México, D.F.: Mc. Graw-Hill

Infobae América. (2013, diciembre 2). Informe PISA: América Latina retrocede en comprensión de lectura, matemática y ciencias. Recuperado de http://www.infobae. com/2013/12/02/1527987-informe-pisa-america-latina-retrocede-comprension-lectura-matematica-y-ciencias/

Jame, G., Zabal, M. y Minguillon, E. (2013). Autoaprendizaje de las matemáticas con nuevas tecnologías en los grados de carácter económico. Revista electrónica sobre la enseńanza de la Economía Pública, 13, 1-22. Recuperado de: http://e-publica.unizar.es//wp-content/uploads/2015/09/ 131JARNE.pdf

Maz-Machado, A. (2012). TIC y matemáticas: una integración en continuo progreso. Revista de Educación Mediatica yTIC, 1 (2), 4-6. Recuperado de: https://doi.org/10.21071/ edmetic.v1i2.2848

Márquez, G. y Sánchez, M. (2010). Motivación al logro, uso de tecnología y aprendizaje estudiantil en matemática del primer ańo de educación superior. Revista Iberoamericana de Educación, 52 (4), 1-11.

Mejía, E. (2005a). Metodología de la investigación científica. Lima: Ediciones de la Universidad Nacional Mayor de San Marcos.

Mejía, E. (2005b). Técnicas e instrumentos de investigación. Lima: Ediciones de la Universidad Nacional Mayor de San Marcos.

Ministerio de Educación. (2005). Evaluación de los aprendizajes de los estudiantes en la educación básica regular. (Directiva No. 004-VMGP-2005, aprobado por R.M. No. 0234-2005-ED) Recuperado de:

http://www.minedu.gob.pe/normatividad/directivas/DIR004-2005-VMGP.pdf

Ministerio de Educación. (2009). Diseño curricular nacional de educación básica regular. Recuperado de: http://www. minedu.gob.pe/DeInteres/xtras/dcn_2009.pdf
Ministerio de Educación. (2015). Rutas del aprendizaje. Versión 2015, ¿Qué y cómo aprenden nuestros niños y niñas? IV Ciclo, Área curricular Matemática. Recuperado de: http://www.minedu.gob.pe/rutas-del-aprendizaje/documentos/Primaria/Matematica-III.pdf

Parra, O. y Díaz, V. (2014). Didáctica de las matemáticas y tecnologías de la información y la comunicación. Revista Educación y Desarrollo Social, 8 (2), 60-81.

Riveros, V., Mendoza, M. y Castro, R. (2011). Las tecnologías de la información y la comunicación en el proceso de instrucción de la matemática. Quórum Académico, 8 (15) 11-130.

Sánchez, H. y Reyes, C. (2006). Metodología y diseños en la Investigación Científica. Lima: Visión Universitaria.

Sarmiento, M. (2007). La enseñanza de las matemáticas y las NTIC. Una estrategia de formación permanente. (Tesis doctoral inédita, Universitat Rovira I Virgili). Recuperada de http://www.tdx.cat/bitstream/handle/10803/8927/C-TESIS_CAPITULO_1.pdf; jsessionid=AC50110E7C3A35E48A72D3B03E0AFA79? sequence=3

UNESCO. (2013). Enfoques estratégicos sobre las TICs en educación en América Latina y el Caribe.

Vidaurre, W. y Vallejos, L. (2015). Software educativo para lograr aprendizajes significativos en el área de matemática. UCV-HACER, 4 (2) 38-45.

Villarreal, M. (2012). Tecnologías y educación matemática: necesidad de nuevos abordajes para la enseñanza. Innovación y Experiencias, 3(5), 73-94. 